\title{
artigo
}

Ribeiro, S.C.; Pires, G.A.R.; Charlo, P.B.; Rodrigues, T.F.C.S.; Paiano, M.; Radovanovic, C.A.T.; Salci, M.A.;

O consumo de derivados do tabaco por adolescentes: Revisão integrativa da literatura

\section{O consumo de derivados do tabaco por adolescentes: Revisão integrativa da literatura}

\begin{abstract}
RESUMO
Objetivou-se identificar na literatura o consumo de derivados do tabaco em adolescentes. Trata-se de Revisão Integrativa, a partir de obras publicadas no período de 2014-2018, na Biblioteca Virtual em Saúde, base eletrônica de dados da Literatura Latino-Americana e do Caribe em Ciências da Saúde, biblioteca digital Scientific Electronic Library Online, Índice Bibliográfico Espanhol de Ciências da Saúde, Science Direct e Literatura Internacional em Ciências da Saúde. Com descritores Decs/Mesh: Adolescente e Derivados do Tabaco integrados pelo conector booleano "and". Na seleção das obras foi utilizado o protocolo para revisões sistemáticas PRISMA Flow Diagram. Para análise adotou-se o software IRAMUTEQ ${ }^{\circledR}$. A amostra foi composta por 60 artigos, variou entre 21 e 109 mil participantes, sendo adolescentes de 9-19 anos. Os principais derivados do tabaco utilizados foram: cigarros eletrônicos, charutos/cigarrilhas, narguilé e cigarro mentolado. Conclui-se que os derivados do tabaco são utilizados por jovens como interação social para manter um "status".
\end{abstract}

DESCRITORES: Derivados do Tabaco; Adolescente; Tabagismo.

\section{ABSTRACT}

The aim was to identify in the literature the consumption of tobacco products in adolescents. This is an Integrative Review, based on works published in the period 2014-2018, in the Virtual Health Library, electronic database of Latin American and Caribbean Health Sciences Literature, digital library Scientific Electronic Library Online, Index Spanish Bibliographic of Health Sciences, Science Direct and International Literature in Health Sciences. With Decs/Mesh descriptors: Adolescent and Tobacco Derivatives integrated by Boolean connector "and". The selection of works used the protocol for systematic reviews PRISMA Flow Diagram. For analysis, IRAMUTEQ ${ }^{\circledR}$ software was adopted. The sample consisted of 60 articles, ranging from 21 to 109 thousand participants, being adolescents from 9-19 years. The main tobacco products used were: electronic cigarettes, cigar/cigarillo, hookah and menthol cigarette. It is concluded that tobacco products are used by young people as social interaction to maintain a status.

KEYWORDS: Tobacco Derivatives; Adolescent; Smoking.

\section{RESUMEN}

El objetivo fue identificar en la literatura el consumo de productos de tabaco en adolescentes. Esta es una Revisión Integrativa, basada en trabajos publicados en el período 2014-2018, en la Biblioteca Virtual en Salud, base de datos electrónica de Literatura en Ciencias de la Salud de América Latina y el Caribe, biblioteca digital Scientific Electronic Library Online, Index Bibliografía española de ciencias de la salud, ciencia directa y literatura internacional en ciencias de la salud, con descriptores Decs / Mesh: adolescentes y derivados del tabaco integrados por el conector booleano " $y$ ". La selección de trabajos utilizó el protocolo para revisiones sistemáticas del diagrama de flujo PRISMA. Para el análisis, se adoptó el software IRAMUTEQ ${ }^{\circledR}$. La muestra constaba de 60 artículos, que iban de 21 a 109 mil participantes, siendo adolescentes de 9 a 19 años. Los principales productos de tabaco utilizados fueron: cigarrillos electrónicos, cigarros / cigarrillos, narguile y cigarrillos mentolados. Se concluye que los jóvenes usan productos de tabaco como interacción social para mantener un estatus.

PALABRAS CLAVE: Derivados del Tabaco; Adolescente; Fumar.

RECEBIDO EM: 04/11/2019 APROVADO EM: 05/11/2019

Sasha Carla Ribeiro

Acadêmica do curso de enfermagem da Universidade Estadual de Maringá (UEM), Maringá, Paraná, Brasil.

Graziele Adrieli Rodrigues Pires

Mestranda no Programa de Enfermagem pela Universidade Estadual de Maringá (UEM). Maringá, Paraná, Brasil. 


\section{Patrícia Bossolani Charlo}

Doutoranda no Programa de Pós Graduação em Enfermagem pela Universidade Estadual de Maringá (UEM). Maringá, Paraná, Brasil.

\section{Thamires Fernandes Cardoso da Silva Rodrigues}

Doutoranda no Programa de Pós Graduação em Enfermagem pela Universidade Estadual de Maringá (UEM). Maringá, Paraná, Brasil.

\section{Marcele Paiano}

Doutora docente do Programa de Pós Graduação em Enfermagem pela Universidade Estadual de Maringá, Maringá, Paraná, Brasil.

\section{Cremilde Aparecida Trindade Radovanovic}

Doutora docente do Programa de Pós Graduação em Enfermagem pela Universidade Estadual de Maringá, Maringá, Paraná, Brasil.

\section{Maria Aparecida Salci}

Doutora docente do Programa de Pós Graduação em Enfermagem pela Universidade Estadual de Maringá, Maringá, Paraná, Brasil.

\section{INTRODUÇÃO}

A s doenças crônicas não transmissíveis (DCNT) representam uma crise global, pelo crescente aumento e por ser responsável por 40 milhões de óbitos por ano no mundo, perfazendo um total de $67 \%$ das mortes nos países de baixa e média renda ${ }^{(1)}$, tendo como principal fator de risco externo o tabagismo. Evidências apontam sobre a necessidade de mudanças no hábito de vida da população urgentemente, pois, no século passado o tabaco foi responsável por 100 milhões de mortes, e está atrelado há previsão de 1 bilhão de óbitos neste século ${ }^{(2)}$.

As crianças, adolescentes e jovens são expostos precocemente a fatores de risco para as DCNT, em especial o tabagismo. Até os 19 anos, a maioria dos fumantes são considerados como dependentes do tabaco. Dessa forma, a Organização Mundial da Saúde (OMS) expande um alerta para o tabagismo como uma doença pediátrica, principalmente ao considerar as estratégicas que a indústria do tabaco e seus derivados desenvolvem para atrair os adolescentes e jovens, repondo diariamente seu mercado de consumidores ${ }^{(3)}$.

Estima-se que existam 1,3 bilhões de pessoas tabagistas no mundo atualmente. A grande maioria destas utiliza cigarros fabricados, os quais representam $92 \%$ do valor da venda de todos os produtos que derivam do tabaco no mundo. Entretanto, devido à conscientização populacional sobre os malefícios do cigarro, a indústria do tabaco vem buscando a diversificação de seus produtos ${ }^{(4,5)}$.
De acordo com a American Câncer Society, no período de 2000 e 2010, as vendas dos diversos produtos derivados do tabaco que não produziam fumaça apresentaram acréscimo em 59\% da renda destinada ao tabaco $^{(5)}$. Os dados da National Youth Tobacco Surveys (NYTS), levantados nos Estados Unidos entre 2011 e 2014 com jovens escolares, apontam o aumento do uso de derivados do tabaco, bem como a diminuição do cigarro convencional ${ }^{(6)}$. Ressalta-se, porém, que todas as formas de tabaco e derivados da nicotina são cancerígenas ${ }^{(4)}$.

Essa transição do cigarro convencional para os derivados de tabaco pode ser fruto da regularização do Programa Nacional de Controle do Tabagismo (PNCT) que, por meio da vinculação com a Atenção Primária à Saúde, obteve uma redução significativa de adultos tabagistas de 11,3\% em 2013, $10,1 \%$ em 2017 para $9,3 \%$ em $2018^{(7)}$.

O PNCT tem como um de seus objetivos a prevenção do início da utilização de derivados do tabaco entre crianças e adolescentes ${ }^{(8)}$. Assim, é de suma importância averiguar na literatura sobre o consumo de derivados de tabaco na população jovem. Nessa perspectiva, emergiu a seguinte questão norteadora: $\mathrm{O}$ que a literatura traz a respeito do consumo de derivados de tabaco entre os adolescentes? Dessa forma, o objetivo do estudo foi identificar na literatura o consumo de derivados do tabaco em adolescentes.

\section{METODOLOGIA}

Trata-se de uma Revisão Integrativa da
Literatura. Tal método permite sumarizar pesquisas primárias realizadas anteriormente e, a partir da avaliação crítica dos resultados, apresentam o estado da arte, contribuindo para o desenvolvimento teórico e, consequente, implicação direta ou indiretamente para a prática assistencial e políticas públicas $^{(9,10)}$.

A presente revisão foi desenvolvida respeitando-se as seguintes fases: 1) formulação do problema; 2) coleta de dados; 3) avaliação dos dados; 4) análise dos dados; e 5) apresentação e interpretação dos resultados $^{(10)}$. O problema formulado desenvolvido na primeira fase consiste na necessidade de se adquirir os conhecimentos disponíveis na literatura sobre o consumo de derivados do tabaco entre os adolescentes brasileiros.

A coleta de dados ocorreu na segunda fase entre os meses de março a junho de 2019 , na qual foi realizado um levantamento na Biblioteca Virtual em Saúde (BVS), base eletrônica de dados da Literatura Latino-Americana e do Caribe em Ciências da Saúde (LILACS), biblioteca digital Scientific Electronic Library Online (SciELO), Índice Bibliográfico Espanhol de Ciências da Saúde (Ibecs), Science Direct e Literatura Internacional em Ciências da Saúde (Medline). Utilizou-se os descritores Decs/Mesh: "Adolescente (Adolescent)" e "Derivados do Tabaco (Tobacco Products)" integrados pelo conector booleano "and", em todas as estratégias de busca.

Os critérios de inclusão foram: artigos originais, publicados na íntegra e dispo- 


\section{artigo}

Ribeiro, S.C.; Pires, G.A.R.; Charlo, P.B.; Rodrigues, T.F.C.S.; Paiano, M.; Radovanovic, C.A.T.; Salci, M.A.;

O consumo de derivados do tabaco por adolescentes: Revisão integrativa da literatura

níveis eletronicamente, divulgados entre janeiro de 2014 a dezembro de 2018, nos idiomas português, inglês ou espanhol, independentemente do método utilizado. Quanto à exclusão: revisões de literatura/reflexão, editoriais, resumos de anais, teses, dissertações, Trabalhos de Conclusão de Curso, boletins epidemiológicos, relatórios de gestão, pesquisa desenvolvi$\mathrm{da}$ com animais, documentos oficiais de programas nacionais ou internacionais, livros, que não atenderem ao objetivo desta pesquisa, e os duplicados (artigos que aparecerem em mais de uma base de dados foram contabilizados apenas uma vez).

$\mathrm{Na}$ fase de coleta de dados, realizou-se a leitura de todas as obras. Para extrair as informaçôes, elaborou-se três instrumentos: o primeiro contendo o título, periódico em que a obra foi publicada, ano da publicação, país do estudo e idioma. O segundo continha a caracterização dos estudos selecionados, com os seguintes tópicos: objetivo do estudo, desenho metodológico e principais resultados que enfatizassem o consumo de derivados do tabaco em adolescentes.

Por fim, o último instrumento elaborado continha a caracterização da amostra de cada obra selecionada. Para tanto, utilizou-se os seguintes tópicos: número de participantes de cada estudo, sexo, idade e história tabagia. Para ilustrar o processo de seleção das obras que integrarem esta revisão, utilizou-se uma adaptação do protocolo para revisões sistemáticas PRISMA Flow Diagram ${ }^{(11)}$.

Após o mapeamento das obras, seguiu-se para fase 3, a qual os dados foram avaliados. As informações chave foram extraídas e para a sua operacionalização, adotou-se o software IRAMUTEQ ALFA 2.3.3.1 - acrônimo de R pour les Analyses Multidimensionnelles de Textes et de Question- naires ${ }^{(12)}$, cujos resultados finais foram apresentados por meio da nuvem de palavras.

Tal software consiste em um programa livre que realiza análise lexical de palavras e ancora-se no software $\mathrm{R}$ o que possibilita diferentes processamentos e análises estatísticas das narrativas produzidas ${ }^{(13)}$.

Todo o processo desenvolvido na seleção dos estudos está representado por meio da Figura 1, que apresenta os quatro passos para organização do estudo: identificação, seleção, elegibilidade e inclusão.

\section{RESULTADOS E DISCUSSÃO}

Detectaram-se nas bases 989 obras e, a partir do processo de seleção e aplicação dos critérios de inclusão e exclusão, foram selecionados para a leitura na íntegra 145 artigos, sendo que destes, 60 artigos integraram esta revisão, os quais estão representados na Tabela 1.

\section{Figura 1. Fluxograma de busca e seleção dos estudos. Maringá, PR, Brasil, 2019}

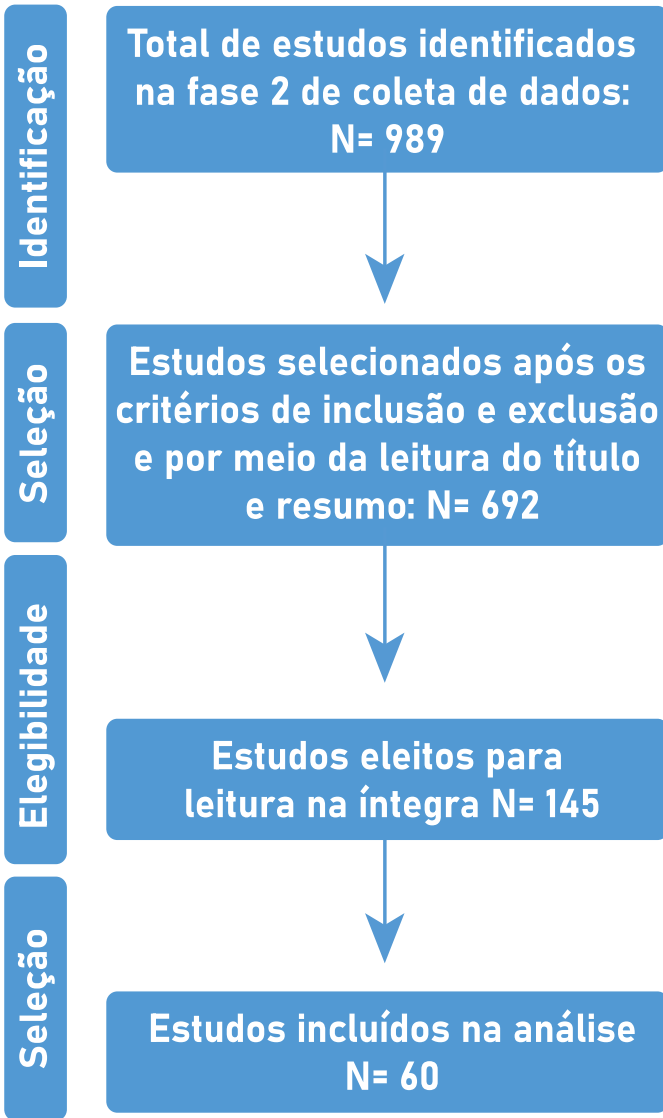

Tabela 1. Identificação dos artigos selecionados, publicados entre 2014 e 2018. Maringá, PR, Brasil, 2019

ID

Frequência de uso entre usuários de produtos de tabaco para

01 estudantes do ensino fundamental e médio - Estados Unidos, 2015-2017

Tendências na facilidade de compra de cigarros entre adoles-

02 centes coreanos: evidências da pesquisa sobre comportamento de risco na Coreia para jovens 2005-2016

\begin{tabular}{lrlr}
\multicolumn{1}{c}{ TÍTULO DO PERIÓDICO } & ANO & PAÍs & IDIOMA \\
$\begin{array}{l}\text { MMWR Morbidity and Mor- } \\
\text { tality Weekly Report }\end{array}$ & 2018 & $\begin{array}{l}\text { Estados } \\
\text { Unidos }\end{array}$ & Inglês \\
BMC Public Health & 2018 & Coreia & Inglês
\end{tabular}


Adolescentes de produtos duais: Aquisição e uso situacional de cigarros e charutos

Mais da metade dos adolescentes usuários de cigarros eletrônicos

04 nunca fumaram um cigarro: resultados de um estudo com crianças em idade escolar no Reino Unido.

Impacto das políticas de controle do tabagismo sobre o tabagismo entre adolescentes sem fumaça e o uso de charutos: uma abordagem de diferenças-em-diferenças

Percepções Favoráveis de Cigarros Eletrônicos Relativos a Cigarros e as Associações com Susceptibilidade ao Uso de Cigarros Eletrônicos em Adolescentes Chineses de Hong Kong

07 Status de peso e uso de cigarros e cigarros eletrônicos em adolescentes

Associações de Concentração de Nicotina de Cigarro Eletrônico

Com os níveis subsequentes de tabagismo e vaping em adolescentes

A idade precoce de início do uso do cigarro eletrônico média a

09 associação entre a impulsividade e a frequência de uso de cigarros eletrônicos em jovens.

Suscetibilidade ao Uso de Produtos de Tabaco entre Jovens na Onda 1 do Estudo de Avaliação da População de Tabaco e Saúde (PATH)

Uso de tabaco em adolescentes em áreas urbanas versus áreas rurais

14 dos Estados Unidos: a influência de ambientes de políticas de controle do tabagismo

Engajamento com o Marketing e as Associações de Tabaco Online com

15 o Uso de Produtos de Tabaco entre os Jovens dos EUA: Resultados da Onda 1 da Avaliação da População do Tabaco e Estudo de Saúde

A Relação entre Frequência de Uso de Cigarro Eletrônico e Frequência e Intensidade do Tabagismo entre Adolescentes Sul-Coreanos

Uso de cigarros eletrônicos não está associado à cessação de cigarros convencionais em jovens fumantes

18 E-cigarros e "gotejamento" entre os jovens do ensino médio

19 Experiências subjetivas no primeiro uso de cigarro, e-cigarros, narguilé e produtos de charuto entre os adolescentes do Texas.

Integrando o impacto do consumo de tabaco de cigarro e narguilé

20 entre adolescentes na região do Mediterrâneo Oriental: um modelo transversal de exposição a substâncias tóxicas em nivel de população

Frequência de padrões de uso de cigarros e cigarros juvenis nos Esta-

21 dos Unidos: a precisão da mensuração é fundamental para informar a saúde pública

Suscetibilidade ao cigarro eletrônico como preditor da iniciação juvenil de cigarros eletrônicos.
Estudo longitudinal do uso de cigarro eletrônico e início do tabagismo entre estudantes do ensino médio no Havaí
Drug and Alcohol

Dependence

Public Health

BMC Public Health

Unidos

Ingles

International Journal of

Environmental Research

and Public Health

American Journal of

Preventive Medicine.

JAMA Pediatrics

ELSEVIER

2017 EUA

Inglês

Caderno de Saúde Pública

2017 Brasil

Português

ELSEVIER

2017 EUA

Inglês

Substance use \& Misuse

2017 EUA

Inglês

Preventive medicine

2017 EUA

Inglês

ELSEVIER

2017 EUA

Inglês

ELSEVIER

2017 EUA

Inglês

Internacional de pesquisa

ambiental e Saúde Pública

2017 Coréia

Inglês

Pediatric Research

2017 EUA

Inglês

PEDIATRICS

2017 EUA

Inglês

Drug and Alcohol

Dependence

2017 EUA

Inglês

BMJ

2016 EUA

Inglês

Nicotine \& Tobacco

Research

2017 EUA

Inglês

Nicotine \& Tobacco

Research

2017 EUA

Inglês

BMJ

2017 EUA

Inglês 


\section{artigo}

Ribeiro, S.C.; Pires, G.A.R.; Charlo, P.B.; Rodrigues, T.F.C.S.; Paiano, M.; Radovanovic, C.A.T.; Salci, M.A.;

O consumo de derivados do tabaco por adolescentes: Revisão integrativa da literatura

24 Prevalência e crenças sobre cigarros eletrônicos e narguilé entre estudantes do ensino médio com asma.

Use of electronic cigarettes among secondary and high school students from a socially disadvantaged rural area in Poland

E-cigarette use is differentially related to smoking onset among lower risk adolescents

27 E-cigarettes, Cigarettes, and the prevalence of adolescent tobacco use

Frequency of Tobacco Use Among Middle and High School Students — United States, 2014

Adolescent and Young Adult Perceptions of Hookah and Little Cigars/ Cigarillos: Implications for Risk Messages

Adolescents' Perceptions of Health Risks, Social Risks, and Benefits Differ Across Tobacco Products

Harm Reduction or Harm Introduction? Prevalence and Correlates of E-Cigarette Use Among French Adolescents

Prevalence and Correlates of E-Cigarette Perceptions and Trial Among Early Adolescents in Mexico

Latent class analysis of current e-cigarette and other substance use in high school students

Use of electronic cigarettes and alternative tobacco products among Romanian adolescentes

Multiple tobacco product use among US adolescents and young adults Dual use of electronic and tobacco cigarettes among adolescents: a cross-sectional study in Poland

Individual, Parental, and Environmental Correlates of Cigar, Cigarillo, and Little Cigar Use Among Middle School Adolescents

Associations of Adolescents' Cigarette, Waterpipe, and Dual Tobacco Use with Parental Tobacco Use

Reporting of cigar use among adolescent tobacco smokers

Poly-Tobacco Use among High School Students

Flavored Tobacco Product Use Among Middle and High School Students - United States, 2014

Uso de produtos de tabaco com sabor entre os jovens dos EUA Idade entre os 12 e os 17 anos, 2013-2014

Associação do Uso de Cigarros Eletrônicos com o Início do Fumar Combustivel do Tabaco na Adolescência Precoce

Cigarro Eletrônico e Uso Tradicional de Cigarros entre Alunos do Ensino Fundamental e Médio na Flórida, 2011-2014

Cachimbo D'agua uma porta de entrada para a iniciação do tabagismo entre adolescentes em Irbid, na Jordânia: um estudo longitudinal

Fatores de risco para cigarro eletrônico, cigarro convencional e uso duplo em adolescentes alemães: um estudo de coorte.
Annals of Epidemiology

2016

EUA

Inglês

BMC Public Health

2016

Polônia

Inglês

Tob Control

2016 EUA

Inglês

Pediatrics

Journal of Health

Communication

2016 EUA

Inglês

2016 EUA

Inglês

Journal of Adolescent Health

2016 EUA

Inglês

Journal of Adolescent Health

2016

França

Inglês

Journal of Adolescent Health

2016

México

Inglês

Drug and Alcohol

Dependence

2016 EUA

Inglês

Int J Public Health

2015

Romênia Inglês

Tob Control

2016 EUA

Inglês

Int J Public Health

2016

Polônia

Inglês

Nicotine \& Tobacco

Research,

2016 EUA

Inglês

Nicotine \& Tobacco

Research,

Addictive Behaviors

2015 Arábia

Inglês

2016 EUA

Inglês

Int. J. Environ. Res. Public

Health

2015 EUA

Inglês

Centers for Disease Control and Prevention MMWR

Morbidity and Mortality

2015 EUA

Inglês

Weekly Report

Centers for Disease Control and Prevention MMWR

Morbidity and Mortality

2015 EUA

Inglês

Weekly Report

JAMA

2015 EUA

Inglês

JAMA

2015 EUA

Inglês

Research Article

2015 EUA

Inglês

Ingenta connect

2015 Jordânia

Inglês

Elsevier

2015

Alema- Inglês
nha


A influência da atividade física no tabagismo entre adolescentes:

evidências da adição de saúde

Determinantes da iniciação ao tabagismo em escolares jordanianos: análise longitudinal.

Uso de narguilé e suscetibilidade cognitiva ao tabagismo entre jovens

49 jordanianos que não fumam cigarro: análise do Global Youth Tobacco Survey 2009.

50 Senso de Coerência e Mitos do Uso do Tabaco entre Adolescentes como Preditores do Uso de Cigarros para Jovens em Situação de Risco

Conscientização e determinantes do consumo de cigarro eletrônico

51 entre adolescentes finlandesas em 2013: um estudo de base populacional

Aumento no uso de cigarros eletrônicos entre adolescentes na Polônia

Produto alternativo de tabaco e uso de drogas entre adolescentes que usam cigarros eletrônicos, cigarros e nunca fumantes.

54 Uso adolescente de produtos de tabaco aromatizado na cidade de Nova York

Intensidade do tabagismo e intenção de continuar a fumar entre os adolescentes fumantes mentolados e não mentolados no Canadá.

Uso de tabaco aromatizado entre estudantes canadenses do $9^{\circ}$ ao

$562^{\circ}$ anos: prevalência e padrões da pesquisa de fumo entre jovens de 2010-2011.

Prevalência e correlatos do uso de narguilé, cigarro, álcool e drogas

57 ao longo da vida entre estudantes do ensino médio em Stoke-on-Trent, Reino Unido: uma análise transversal.
Nicotine \& Tobacco

tal e médio dos EUA

Percepções dos adolescentes sobre os riscos e benefícios dos ci-

60 qualitativa

Research

Nicotine \& Tobacco

Research

ResearchGate

HHS Public Access

Tobacco control

Elsevier

Nicotine \& Tobacco

Research

Original paper

Original Research

Journal of public health

Research Report

Elsevier

2015 EUA

Inglês

2015 Jordânia Inglês

2015 Jordânia Inglês

2015 EUA

Inglês

2015 Finlândia Inglês

2014 Polônia Inglês

2014 EUA Inglês

2014 EUA Inglês

2014 Canadá Inglês

2014 Canadá Inglês

2014 Reino Inglês

2014 Noruega Inglês

2014 EUA

Inglês

Elsevier
A partir da organização do software, o corpus textual apresentou 238 segmentos de textos, com aproveitamento de $72 \%$.
$\mathrm{Da}$ nuvem, evidenciaram-se os vocábulos: Cigarro $(n=463)$, Tabaco $(n=196)$, Eletrônico (cigarro eletrônico) $(n=120)$,
Charuto ( $\mathrm{n}=108)$, Fumante $(\mathrm{n}=79)$, Cigarrilha $(n=22)$ e Nicotina $(n=17)$, conforme Figura 2. 


\section{artigo}

Ribeiro, S.C.; Pires, G.A.R.; Charlo, P.B.; Rodrigues, T.F.C.S.; Paiano, M.; Radovanovic, C.A.T.; Salci, M.A.;

O consumo de derivados do tabaco por adolescentes: Revisão integrativa da literatura

Figura 2. Nuvem de palavras. Maringá, PR, Brasil, 2019.

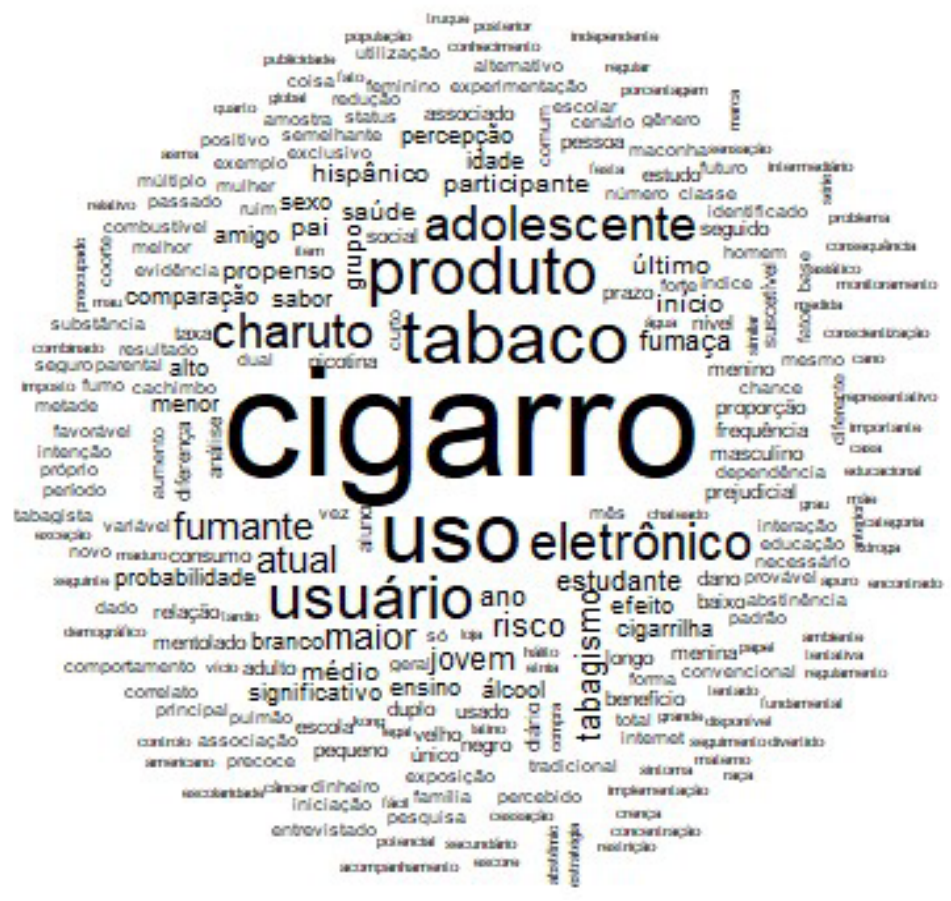

A partir da convergência entre os vocábulos destacados pelo IRAMUTEQ ${ }^{\circ}$ e a leitura crítica do conteúdo, identificou-se que os cigarros eletrônicos, narguilé, charuto/cigarrilha e cigarro mentolado são os produtos que os adolescentes mais consomem e, consequentemente, aumentam a probabilidade de se tornarem tabagistas na vida adulta.

Quando questionados sobre o motivo por escolher tais produtos, muitos adolescentes os compreendem como "menos prejudiciais" que o cigarro comum, bem como por proporcionar "status" tanto no ambiente escolar como entre o grupo de amigos. Não obstante, alguns adolescentes indicaram que iniciaram o consumo de derivados do tabaco a partir da influência de familiares, especialmente pais e mães.

Há inúmeros motivos que impulsionam os adolescentes a preferirem o cigarro eletrônico, destacando-se a diver- sidade de sabores existentes, bem como pelo "status social" que o seu consumo confere aos jovens ${ }^{(14)}$. Estudo ${ }^{(15)}$ realizado na China, identificou que 5,3\% dos jovens percebiam que aqueles que utilizavam o cigarro eletrônico eram pessoas "mais chiques" que as demais, $4,8 \%$ o preferiam por ser mais fácil para comprar e usá-lo sem ser notado, e $12,4 \%$ declaram que o seu uso torna o ambiente mais amigável.

Constatou que, além do status social conferido pelo uso de cigarro eletrônico, os adolescentes desconheciam os seus malefícios e o apontavam como um produto menos prejudicial do que os cigarros convencionais. Esses dados corroboram com a pesquisa realizada em Hong Kong - China ${ }^{(15)}$, o qual $25,2 \%$ dos participantes relatavam que os cigarros eletrônicos eram menos propensos a causar acidentes como incêndios e queimaduras e $24,5 \%$ menos prejudiciais para os usuários. Tal escolha, impulsionou a experimentação precoce de tabaco além de propiciar o tabagismo na vida adulta. Nesse contexto, faz-se importante alertar os adolescentes sobre as reais consequências sobre o cigarro eletrônico e outros derivados do tabaco, que estão popularmente disseminados entre os jovens, no intuito de diminuir o índice de usuários nessa faixa etária ${ }^{(16)}$.

Os achados desta revisão corroboram a literatura, cujos dados da Pesquisa Nacional de Saúde do Escolar, apontam que o uso de derivados do tabaco entre adolescentes passou de 4,8\% em 2012, para $6,1 \%$ em $2015^{(17)}$. No que tange à influência do meio social para a iniciação tabágica na adolescência, estudo $^{(18)}$ brasileiro identificou que o uso de tabaco se associa ao desejo de sentir-se como parte de um determinado grupo, de maneira que muitos participantes ressaltaram que "apenas os alunos legais da escola utilizam tabaco". 
Em relação à influência familiar na iniciação precoce do tabagismo, estudo ${ }^{(19)}$ conduzido com 6.264 pessoas, com idade entre 14 e 19 anos, notou que quando um dos pais, ou ambos, são tabagistas, há maior chance de os adolescentes tornarem-se futuros fumantes, quando comparado àqueles que não possuíam pais dependentes de tabaco. Os pais atuam como modelos para os filhos e, influenciam suas opiniões, a presença de comportamentos aditivos eleva a probabilidade de seus descentes também se tornarem tabagistas.

\section{CONCLUSÃO}

A partir dos resultados, pode-se identificar que os principais produtos derivados do tabaco consumidos por adolescentes são: cigarro eletrônico, charuto/cigarrilha, narguilé e cigarro mentolado; esses derivados eram utilizados pela maioria dos adolescentes para inclusão em meio social, se enquadrando assim em padróes estipulados pelos próprios jovens, dessa forma, mantinham um "status" e idealização de que os derivados iriam trazer níveis reduzidos de malefícios à saúde. $\mathrm{O}$ ta- bagismo familiar também é um fator preditivo da iniciação do cigarro, favorecendo o consumo na vida adulta.

É importante salientar que os profissionais de saúde devem abordar o tema nas intervenções de saúde direcionadas às crianças e aos adolescentes, no intuito de desmistificar questões sobre os produtos derivados do tabaco, tão populares entre esse público e, assim, desenvolver ações para a prevenção da experimentação precoce e ressaltar também a importância de direcionar para a família as ações de promoção da saúde.

\section{REFERÊNCIAS}

1. World Health Organization. WHO report on the global tobacco epidemic [Internet]. 2017 [access 11 Abr. 2018]. Available from: http://www.who.int/tobacco/global_report/en/.

2. Jha P, Peto R. Global effects of smoking, of quitting, and of taking tobacco. N Engl J Med. 2014; 370:60-8.

3. Instituto Nacional de Câncer (BR). Ações e Programas no Brasil - Programa Nacional de Controle do Tabagismo [acesso em 20 out 2018]. Disponivel em:

http://www2.inca.gov.br/wps/wcm/connect/acoes_programas/site/home/nobrasil/programa-nacional-controle-tabagismo/criancas-adolescentes-jovens. 2018.

4. Jemal A, Vineis P, Bray F, Torre L, Forman D. The Cancer Atlas. Second Ed. Atlanta, GA: American Cancer Society; 2014

5. Instituto Nacional de Câncer (BR). Cigarros eletrônicos: o que sabemos? Estudo sobre a composição do vapor e danos à saúde, o papel na redução de danos e no tratamento da dependência de nicotina / Instituto Nacional de Câncer José Alencar Gomes da Silva; organização Stella Regina Martins. - Rio de Janeiro: INCA, 2016.

6. Arrazola RA, et al. Tobacco use among middle and righ school students - United States, 2011-2014. MMWR Morb Mortal Wkly Rep 2015; 64:381-5.

7. Hallal AL. et al. Associação entre a exposição a maços de cigarros em pontos de venda e susceptibilidade ao tabagismo entre adolescentes brasileiros. Jornal Brasileiro de Pneumologia. 2018; 44(1):49-51.

8. Campos P, Gomide M. O Programa Nacional de Controle do Tabagismo (PNCT) na perspectiva social: a análise de redes, capital e apoio social. Cadernos Saúde Coletiva. 2015; 23(4):436444.

9. Mendes KDS, Silveira RCCP, Galvão CM. Revisão integrativa: método de pesquisa para a incorporação de evidências na saúde e na enfermagem. Texto \& Contexto de Enfermagem. 2008;

\section{7(4):758-764.}

10. Whittemore $\mathbf{R}$, Knalf $\mathbf{K}$. The integrative review: updated methodology. Journal of Advanced Nursing. 2005; 52(5):546553.

11. Moher D, et al. Preferred reporting items for systematic review and meta-analysis protocols (PRISMA-P). PLoS Medicine. 2009; 6(7):2009.

12. Jesus GJ, et al. Difficulties of living with HIV/Aids: obstacles to quality of life. Acta Paul Enfermagem. 2017; 30(3):301-7.

13. Camargo VP, Quintana AM, Weisshimer TKS, Junges $N$, Martins BMC. Transplante renal: um caminho para a vida ou um passo para a morte? Revista Contexto e Saúde. 2013; 10(20):515-524.

14. Pereira UM, Solé D. Cigarros eletrônicos: esses ilustres desconhecidos. Arq Asma Alerg Imunol. 2018; 2(3):214-245

15. Leung LT. et al. Favourable Perceptions of Electronic $\mathbf{C i}-$ garettes Relative to Cigarettes and the Associations with Susceptibility to Electronic Cigarette Use in Hong Kong Chinese Adolescents. Int. J. Environ. Res. Public Health 2018; 15(54):345-60.

16. Roditis ML, Felsher BH. Adolescents' Perceptions of Risks and Benefits of Conventional Cigarettes, E-cigarettes, and Marijuana: A Qualitative Analysis. Journal of Adolescent Health. 2015; 57:179-85.

17. Malta $D C$, et al. Fatores associados ao uso de narguilé e outros produtos do tabaco entre escolares. Revista brasileira de Epidemiologia. 2018; 21(1):135-49.

18. Reinaldo MAS, Pereira MO. Fatores associados ao tabagismo entre adolescentes do sexo feminino. Saúde debate. 2018; 42(4):156-165.

19. Oliveira LM, et al. Influência do tabagismo parental no consumo de álcool e drogas ilícitas entre adolescentes. Einstein (São Paulo). 2019 ;17(1):eA04377. 